\title{
MONITORAMENTO TELEFÔNICO COMO ESTRATEGIA EDUCATIVA PARA O AUTOCUIDADO DAS PESSOAS COM DIABETES NA ATENÇÃO PRIMÁRIA
}

\author{
TELEPHONE-BASED MONITORING: AN EDUCATIONAL STRATEGY \\ FOR DIABETES SELF-CARE AT THE PRIMARY HEALTH CARE LEVEL
}

\section{MONITOREO TELEFÓNICO COMO ESTRATEGIA EDUCATIVA PARA EL AUTOCUIDADO DE PERSONAS CON DIABETES EN ATENCIÓN PRIMARIA}

\author{
Heloisa Carvalho Torres* \\ ILKa Afonso ReIs ${ }^{* *}$ \\ Carolina Roque ${ }^{* * *}$ \\ PRISCILA FARIA ${ }^{* * * *}$
}

\begin{abstract}
RESUMO
Objetivos: Descrever a experiência do monitoramento telefônico como estratégia educativa na educação do autocuidado da pessoa com diabetes na atenção primária. Material e método: Trata-se de um estudo descritivo, realizado com 115 usuários com diabetes residentes em domicílios com linha telefônica fixa e em seguimento no programa educativo na atenção primária, ano de 2010. As entrevistas telefônicas seguiram um roteiro semiestruturado. Resultados: Dos 115 entrevistados, 44,8\% praticam atividade física e 58,3\% seguem a dieta. Em ambos os grupos, a maioria dos praticantes de atividade física são adeptos da caminhada. O percentual de usuários que seguem a dieta é praticamente o mesmo entre homens e mulheres (60,0 e 57,9\%). Conclusão: O monitoramento permitiu reforçar a comunicação e as informações para a educação dos usuários com diabetes, fortaleceu o vínculo entre usuários e profissionais e possibilitou que os profissionais interviessem junto às dificuldades dos usuários no manejo da doença, de forma rápida e efetiva.
\end{abstract}

Palavras chave: Sistema de vigilância por inquérito telefônico, comunicação em saúde, diabetes mellitus, autocuidado, educação em saúde.

\footnotetext{
ABSTRACT

Objective: to describe the experience of telephone-based monitoring as an educational strategy to teach selfcare to people with diabetes at the primary health care level. Material and method: This descriptive study was performed with 115 clients with diabetes who had a landline telephone at home and were being followed in a primary health care educational program in 2010. The telephone interviews were performed following a semi-

* Pós Doutora, professora do Departamento de Enfermagem Aplicada da Escola de Enfermagem da Universidade Federal de Minas Gerais- EE/UFMG- Belo Horizonte (MG), Brasil. E-mail: heloisa.ufmg@gmail.com

${ }^{* *}$ Doutora, professora do Departamento de Estatística da Universidade Federal de Minas Gerais - UFMG- Belo Horizonte (MG), Brasil. E-mail: ilka@est.ufmg.br

${ }^{* * *}$ Bolsista de Iniciação Cientifica; aluna do $9^{\circ}$ período do Curso de Graduação da Escola de Enfermagem da Universidade Federal de Minas Gerais - UFMG- Belo Horizonte (MG), Brasil. E-mail: carolina_roque@yahoo.com.br

${ }^{* * * *}$ Bolsista de Iniciação Cientifica; aluna do 9o período do Curso de Graduação da Escola de Enfermagem da Universidade Federal de Minas Gerais - UFMG- Belo Horizonte (MG), Brasil. E-mail: priscila@yahoo.com.br
} 
-structured script. Results: From the 115 interviewed clients, $44.8 \%$ were physically active and $58.3 \%$ followed a diet. In both groups, most of those engaged in physical activity reported walking. Basically the same percentage was found for male and female clients who followed their diet (60.0\% and $57.9 \%$, respectively). Conclusion: Monitoring allowed reinforcing communication and the information required for the education of clients with diabetes, it strengthened the bond between clients and professionals, and permitted the professionals to intervene on the difficulties that the clients presented regarding their management of the diseases, in a fast and effective way.

Key words: Telephone-based surveillance system, health communication, diabetes mellitus, self care, health education

\section{RESUMEN}

Objetivo: Describir la experiencia del monitoreo telefónico como estrategia educativa en enseñanza del autocuidado de personas con diabetes en la atención primaria. Material y método: Estudio descriptivo, realizado con 115 pacientes de diabetes residentes en domicilios con línea telefónica fija y en seguimiento educativo en la atención primaria, año 2010. Entrevistas telefónicas fueron realizadas según rutina semiestructurada. Resultados: De los 115 entrevistados, 44,8\% practican actividad física y 58,3\% siguen la dieta. En ambos grupos, la mayoría de practicantes de actividad física son adeptos a las caminatas. El porcentaje de pacientes que sigue la dieta es casi el mismo entre hombres y mujeres (60,0 y 57,9\%). Conclusión: el monitoreo permitió reforzar la comunicación y las informaciones para educar a los pacientes con diabetes, fortaleció el vínculo entre pacientes y profesionales y posibilitó que éstos intervinieran ante las dificultades de los pacientes en el manejo de la enfermedad, de forma rápida y efectiva.

Palabras clave: Sistema de vigilancia por encuesta telefónica, comunicación en salud, diabetes mellitus, autocuidado, educación en salud.

Fecha recepción: 02/10/11 Fecha aceptación: 27/03/13

\section{INTRODUÇÃO}

Segundo a Organização Mundial de Saúde (OMS), as doenças crônicas não transmissíveis (doenças cardiovasculares, câncer, diabetes e outras) já contabilizam $58,5 \%$ de todas as mortes ao redor do mundo e correspondem por $45,9 \%$ das doenças globais. No Brasil, estas doenças também são de grande magnitude e correspondem a mais de $60 \%$ dos óbitos e tem grande importância na morbidade ambulatorial e hospitalar $(1,2)$.

Dentre os usuários atendidos na atenção primária e com grande demanda para o sistema de saúde, destacam-se os usuários com hipertensão arterial sistêmica e diabetes mellitus presentes em 23,1 e 5,2\% da população adulta brasileiros, respectivamente, segundo dados auto referidos do VIGITEL (Vigilância de Fatores de Risco e Proteção para Doenças crônicas por Inquérito Telefônico) (2). Tais agravos favorecem a redução da expectativa e da qualidade de vida desta população denotando a necessidade de estratégias de promoção, prevenção e controle, em especial o diabetes.

Torna-se importante o tratamento precoce e o incentivo ao autocuidado da doença, em função das complicações, co-morbidades associadas e pelo aumento crescente das taxas se mortalidade por diabetes (2). Neste estudo assume-se a educação para o autocuidado em diabetes, definida como um plano de ações estruturado para fornecer ao usuário, conhecimentos, atitudes e habilida- 
des necessárias para: a) o desempenho do autogerenciamento dos cuidados da doença no controle das crises (hipoglicemia; hiperglicemia) e b) para a mudança de comportamento, especialmente dentro das áreas de nutrição e atividade física. O objetivo do autocuidado é capacitar o usuário a entender e a motivar-se a participar efetivamente do regime terapêutico no dia-a-dia (3).

A maior parte da educação do autocuidado em diabetes ocorre fora do serviço de saúde, enquanto as pessoas estão vivendo suas vidas em casa e na comunidade. É freqüentemente encontrada no acompanhamento dos usuários no processo educativo e como método auxiliar nas intervenções realizadas para autocuidado da doença $(3,4)$.

A literatura (4-7) reconhece que é necessário monitorar e acompanhar prática do autocuidado dos usuários, proporcionando conhecimentos atualizados sobre a doença, comunicação efetiva, escuta e compreensão, bem como a capacidade de negociação com os indivíduos visando à redução das barreiras ao atendimento de qualidade para alcançar a educação em saúde de forma efetiva em diabetes. $\mathrm{O}$ telefone pode ser uma opção viável para este monitoramento dos usuários com diabetes, como uma forma de encurtar distâncias não apenas físicas, mas também sócio-culturais, uma vez que se considera o contexto do indivíduo ao se expressar pelo telefone, seja pela sua entonação de voz, seu silêncio, pelo conteúdo da conversa exposta, dentre outros parâmetros $(2,8)$.

Trata-se de uma tecnologia inovadora de informação e comunicação pautada em uma abordagem construtivista, a partir de uma reflexão crítica da realidade em que se consideram a autonomia, os valores, as opiniões e as experiências do usuário, tornando-o responsável pela manutenção de sua saúde e adesão às práticas de autocuidado da doença (7). O monitoramento telefônico é considerado, portanto, uma forma de comunicação efetiva de escuta e compreensão por parte do profissional de saúde e do usuário (8-10).
Os profissionais de saúde da atenção primária e a comunidade acadêmica universitária envolvida com a prática dos serviços de saúde, ao delinearem o programa de educação em diabetes, defrontaram-se com a necessidade de monitorar os usuários com diabetes, buscando transmitir as informações sobre o autocuidado, de forma clara e objetiva, procurando detectar as inquietações dos usuários frente às dificuldades e barreiras para a realização da dieta e a prática de exercícios. Diante desse cenário, entende-se como viável a proposta de monitoramento dos usuários com diabetes, a partir do uso do telefone, assumindo o conceito de monitoramento telefônico como acompanhamento contínuo, regular e sistemático do desenvolvimento dessa estratégia em relação a seus objetivos e metas para as praticas de autocuidado associado à dieta e atividade física.

Este estudo descreve a experiência do monitoramento telefônico como estratégia educativa na educação do autocuidado da pessoa com diabetes na atenção primária.

\section{MATERIAL E MÉTODO}

Trata-se de um estudo descritivo, realizado com 115 usuários com diabetes mellitus tipo 2 em seguimento no Programa Educativo desenvolvido na Atenção primária, no ano de 2010 em quatro Unidade de Saúde de Belo Horizonte. O estudo foi conduzido por docentes e professores da Escola de Enfermagem da UFMG, no projeto "Avaliação das ações de promoção da saúde em diabetes tipo 2", em parceria com a Secretaria Municipal de Saúde de Belo Horizonte e compõe um programa educativo junto aos usuários cadastrados com diabetes.

O programa está direcionado à educação dos usuários com diabetes tipo 2, cadastrados nas quatro Unidade de Saúde e visa fortalecer o componente educativo do autocuidados, o qual envolve a dieta, a atividade 
física, a medicação e o monitoramento da glicose. O processo educativo foi constituído por um conjunto de intervenções, voltadas para o conceito da doença, os sinais e sintomas e suas complicações, os princípios da dieta, a prática de atividade física e os aspectos psicológicos relacionados à mudança de comportamento para o controle da doença. As intervenções foram realizadas em sessões de grupos, a cada três meses, com duração de duas horas, permitindo um aprofundamento das discussões. Nos intervalos das sessões educativas, os usuários foram monitorados por telefone.

\section{Estratégia Educativa: Monitoramento Telefônico}

O monitoramento telefônico como estratégia educativa para as praticas de autocuidado, relacionada aos conhecimentos e à mudança voluntária de comportamento associado a dieta e atividade física, cuja finalidade foi favorecer a motivação dos usuários, encorajamento para adesão ao regime terapêutico da doença, acolhimento do usuário, investigação das dificuldades para as práticas de autocuidado, orientação quanto à prevenção das complicações agudas e crônicas da doença e ajudar o usuário a decidir qual a melhor forma de aprimorar o controle metabólico.

$\mathrm{O}$ monitoramento telefônico seguiu um roteiro estruturado, abordando questões relacionadas ao plano alimentar, atividade física, sentimentos, barreiras e metas para as práticas de autocuidado. No decorrer da intervenção os usuários foram questionados sobre as questões descritas abaixo:

- "Qual aspecto do cuidado com o diabetes, você está insatisfeito?",

- "Você consegue colocar em pratica todos os cuidados necessários para o controle da doença?"

- "Você consegue superar as barreiras de cuidado para o diabetes?"

- "Você consegue dizer como se sente sobre

\section{o diabetes?"}

- "Você consegue pensar em maneiras positivas que te motivem a cuidar da doença?" e,

- "Você consegue pedir apoio para cuidar do diabetes quando precisa?"

O monitoramento telefônico foi realizado por quatro alunas bolsistas do curso de graduação em Enfermagem, que foram capacitados e acompanhadas por docentes do Curso de Enfermagem da Universidade Federal de Minas Gerais. Cada bolsista assumiu em média 28 usuários. O monitoramento telefônico foi realizado no período de 09.00 às $11.00 \mathrm{~h}$ e 14.00 às $18.00 \mathrm{~h}$, para evitar os horários de almoço, tele-jornal e novelas. As orientações telefônicas foram estruturadas para serem conduzidos com duração de 15 a 25 min nos intervalos dos grupos operativos, a cada três meses, e para convidar os usuários a participarem do processo de ensino aprendizagem e agendar a marcação dos exames. Foram realizadas em médias oito ligações telefônicas para cada usuário.

Os dados foram registrados na planilha Excel para arquivo do monitoramento telefônico.

Para a coleta dos dados das informações, foi elaborado um protocolo de monitoramento telefônico, que abordava as características sócio-demográfica e econômica dos participantes, diagnóstico e tratamento da doença, hábitos de vida relacionados à alimentação e prática de atividade física, dados antropométricos (peso e índice de massa corporal) e exames de hemoglobinaglicada - HbA1c. Além do diário de campo para as anotações e a descrição da conversa, o horário e a duração da ligação. A análise estatística foi feita por meio de estatísticas descritivas, como distribuições de frequência e medidas-resumo (média e desvio-padrão); para o processamento dos dados utilizou-se o programa do MINITAB $^{\oplus}$.

O projeto foi aceito pelo Comitê de Ética em Pesquisa da Secretaria Estadual de Saúde, 
mediante o parecer $\mathrm{n}^{\circ}$ 0024.040410.203.09 e todos os participantes assinaram o Termo de Consentimento Livre e Esclarecido.

\section{RESULTADOS}

Foram monitorados por telefone 115 usuários com diabetes mellitus. Observou-se no estudo uma população adulta com idade média de 62,6 anos, com maior freqüência de mulheres 80\% (92); baixa escolaridade; glicose sanguínea elevada - HbA1c: 9.08 \%, em média; tempo médio de duração do diabetes: 11 anos, média do Índice de Massa Corporal - IMC: $29,98 \mathrm{~kg} / \mathrm{m}^{2}$, apresentados na (Tabelas 1 e 2).

Em relação à prática de atividade física, apenas $32 \%(n=37)$ praticavam atividade física e $31 \%$ de maneira regular, $30 \mathrm{~min} \mathrm{em}$ três ou mais dias na semana. A prática mais comum foi a da caminhada.

Tabela 1. Distribuição de freqüências de algumas características sócio-demográficas segundo sexo, Unidades de Saúde de Belo Horizonte selecionadas, 2011.

\begin{tabular}{|c|c|c|c|c|}
\hline \multirow{3}{*}{ Característica } & & \multicolumn{2}{|c|}{ Sexo } & \multirow{3}{*}{ Total } \\
\hline & & Masculino & Feminino & \\
\hline & & $\mathbf{n}(\%)$ & $\mathbf{n}(\%)$ & \\
\hline \multirow{8}{*}{ Escolaridade } & Analfabeto & $1(4.4 \%)$ & $7(7.6 \%)$ & $8(7.0 \%)$ \\
\hline & Primário Incompleto & $3(13.0 \%)$ & $8(8.7 \%)$ & $11(9.6 \%)$ \\
\hline & Primário completo & $9(39.0 \%)$ & $47(51.1 \%)$ & $56(48.6 \%)$ \\
\hline & Fundamental incompleto & $3(13.0 \%)$ & $12(13.0 \%)$ & $15(13.0 \%)$ \\
\hline & Fundamental completo & $3(13.0 \%)$ & $7(7.6 \%)$ & $10(8.7 \%)$ \\
\hline & Ensino médio incompleto/completo & $2(8.8 \%)$ & $9(9.8 \%)$ & $11(9.6 \%)$ \\
\hline & Superior incompleto/completo & $2(8.8 \%)$ & $2(2.2 \%)$ & $4(3.5 \%)$ \\
\hline & Total & $23(100 \%)$ & $92(100 \%)$ & $115(100 \%)$ \\
\hline \multirow{7}{*}{ Ocupação } & Aposentado/pensionista & $15(65.3 \%)$ & $44(47.8 \%)$ & $59(51.3 \%)$ \\
\hline & Doméstico/Do lar & $1(4.3 \%)$ & $29(31.5 \%)$ & $30(26.1 \%)$ \\
\hline & Serviços & $5(21.8 \%)$ & $12(13.0 \%)$ & $17(14.8 \%)$ \\
\hline & Comerciário & $1(4.3 \%)$ & $3(3.3 \%)$ & $4(3.5 \%)$ \\
\hline & Diarista/Faxineiro & $0(0 \%)$ & $3(3.3 \%)$ & $3(2.6 \%)$ \\
\hline & Outros & $1(4.3 \%)$ & $1(1.1 \%)$ & $2(1.7 \%)$ \\
\hline & Total & $23(100 \%)$ & $92(100 \%)$ & $115(100 \%)$ \\
\hline \multirow{4}{*}{ Estado Civil } & Solteiro/Divorciado & $2(8.7 \%)$ & $17(18.5 \%)$ & $19(16.5 \%)$ \\
\hline & Casado/Amasiado & $20(87.0 \%)$ & $52(56.5 \%)$ & $72(62.6 \%)$ \\
\hline & Viúvo & $1(4.3 \%)$ & $23(25.0 \%)$ & $24(20.9 \%)$ \\
\hline & Total & $23(100 \%)$ & $92(100 \%)$ & $115(100 \%)$ \\
\hline
\end{tabular}


Tabela 2. Medidas-resumo (média e desvio-padrão) de idade, duração da doença, medidas antropométricas e bioquímicas, segundo sexo, Unidades de Saúde de Belo Horizonte selecionadas, 2011.

\begin{tabular}{|c|c|c|c|c|c|c|}
\hline \multirow{3}{*}{ Característica } & \multicolumn{4}{|c|}{ Sexo } & \multirow{2}{*}{\multicolumn{2}{|c|}{ Total }} \\
\hline & \multicolumn{2}{|c|}{ Masculino } & \multicolumn{2}{|c|}{ Feminino } & & \\
\hline & $\mathbf{n}$ & DP & $\mathbf{n}$ & DP & $\mathbf{n}$ & DP \\
\hline & 62.6 & 12,0 & 62,6 & 14 & 62,6 & 10,6 \\
\hline Idade (anos) & \multicolumn{2}{|c|}{$62.6(12.0)$} & \multicolumn{2}{|c|}{$62.6(14.0)$} & \multicolumn{2}{|c|}{$62.6(10.6)$} \\
\hline Duração da doença (anos) & \multicolumn{2}{|c|}{$11.5(7.4)$} & \multicolumn{2}{|c|}{$11.0(8.7)$} & \multicolumn{2}{|c|}{$11.1(8.4)$} \\
\hline Peso $(\mathrm{kg})$ & \multicolumn{2}{|c|}{$(76.0)(14.3)$} & \multicolumn{2}{|c|}{ (73.1) (13.7) } & \multicolumn{2}{|c|}{$73.7(13.8)$} \\
\hline Altura (m) & \multicolumn{2}{|c|}{$1.68(0.08)$} & \multicolumn{2}{|c|}{$1.54(0.08)$} & \multicolumn{2}{|c|}{$1.57(0.10)$} \\
\hline Circunferência Abdominal $(\mathrm{cm})$ & \multicolumn{2}{|c|}{$109.17(10.19)$} & \multicolumn{2}{|c|}{$103.67(11.09)$} & \multicolumn{2}{|c|}{$104.50(11.58)$} \\
\hline Glicohemoglobina HbAlc & \multicolumn{2}{|c|}{$6.58(3.80)$} & \multicolumn{2}{|c|}{$8.72(2.32)$} & \multicolumn{2}{|c|}{$9.08(1.65)$} \\
\hline Colesterol Total $(\mathrm{mg} / \mathrm{dl})$ & \multicolumn{2}{|c|}{$143.0(18.2)$} & \multicolumn{2}{|c|}{$197.8(38.6)$} & \multicolumn{2}{|c|}{$190.1(42.5)$} \\
\hline Colesterol HDL (mg/dl) & \multicolumn{2}{|c|}{$33.4(8.6)$} & \multicolumn{2}{|c|}{$54.4(29.0)$} & \multicolumn{2}{|c|}{$52.0(28.7)$} \\
\hline Colesterol LDL (mg/dl) & \multicolumn{2}{|c|}{$67.8(18.6)$} & \multicolumn{2}{|c|}{$119.0(39.5)$} & \multicolumn{2}{|c|}{$112.5(42.3)$} \\
\hline Colesterol VLDL (mg/dl) & \multicolumn{2}{|c|}{$35.8(20.3)$} & \multicolumn{2}{|c|}{$30.9(14.9)$} & \multicolumn{2}{|c|}{$31.4(15.8)$} \\
\hline Triglicérides (mg/dl) & \multicolumn{2}{|c|}{$179.5(99.6)$} & \multicolumn{2}{|c|}{$165.0(97.5)$} & \multicolumn{2}{|c|}{$166.9(96.2)$} \\
\hline
\end{tabular}

Tabela 3. Distribuição de freqüências da duração da atividade física e valores médios para freqüência semanal segundo sexo do usuário.

\begin{tabular}{|c|c|c|c|c|c|c|}
\hline \multirow{2}{*}{ Sexo } & \multicolumn{5}{|c|}{ Duração da Atividade Física (minutos) } & \multirow{2}{*}{$\begin{array}{l}\text { Freqüência semanal } \\
\text { média, em dias. } \\
\text { Desvio-padrão entre } \\
\text { parênteses }\end{array}$} \\
\hline & 15 & 30 & 45 & 60 & Total & \\
\hline Masculino & $1(9.1 \%)$ & $6(54.5)$ & $2(18.2)$ & $2(18.2 \%)$ & $11(100 \%)$ & $3.7(2.0)$ \\
\hline Feminino & $0(0.0 \%)$ & $9(34.6 \%)$ & $3(11.5 \%)$ & $14(53.9 \%)$ & $26(100 \%)$ & $4.0(1.6)$ \\
\hline Total & $1(2.7 \%)$ & $15(40.5 \%)$ & $5(13.5 \%)$ & $16(43.3 \%)$ & $37(100 \%)$ & $3.9(1.7)$ \\
\hline
\end{tabular}

Quanto à freqüência das refeições diárias, o número médio de refeições diárias é semelhante entre homens e mulheres (de 4 a 5 refeições). Constatou-se melhora na diminuição de alimentos que contém açúcar e massas, aumento na ingestão de alimentos ricos em fibras e redução do consumo de alimentos gordurosos e de doces.

Entre os usuários do sexo masculino, $89,5 \%$ fazem uso de medicação, percentual que é semelhante entre os usuários do sexo feminino (90\%). 


\section{Experiências e dificuldades para a realização da prática do autocuidado em diabetes}

Os usuários apresentaram-se receptivos por receberem as ligações, sentindo-se acolhidos, motivados ao perceber o interesse por parte dos alunos, incentivando-os a elaborar estratégias de enfretamento da doença.

Observamos no decorrer do monitoramento que algumas dificuldades em relação ao seguimento da dieta foram manifestadas por alguns usuários, como falta de apetite, motivação, tempo, questões econômicas, e em relação á prática de atividade física, foram apontados fatores como: falta de animo, problemas articulares, cardíacos, perda de parentes (Tabela 4). As barreiras para o autocuidado foram trabalhadas com calma e o enfrentamento destas foram adaptadas para cada usuário.

Tabela 4. Distribuição de freqüências das razões para dificuldades no seguimento de dieta e na prática de atividade física, segundo sexo.

\begin{tabular}{|c|c|c|c|c|}
\hline \multirow{7}{*}{$\begin{array}{l}\text { Dificuldades na } \\
\text { dieta }(55 \%)\end{array}$} & & \multicolumn{2}{|c|}{ Sexo } & \multirow{2}{*}{ Tota } \\
\hline & & Masculino & Feminino & \\
\hline & Apetite & 1 & 1 & 2 \\
\hline & Fator econômico & 0 & 1 & 1 \\
\hline & Motivação & 3 & 12 & 15 \\
\hline & Falta de tempo & 0 & 1 & 1 \\
\hline & Total & 4 & 15 & 19 \\
\hline \multirow{6}{*}{$\begin{array}{l}\text { Dificuldades na } \\
\text { prática de atividade } \\
\text { física } \\
(58.6 \%)\end{array}$} & Falta de ânimo/motivação & 1 & 6 & 7 \\
\hline & Perda de ente querido & 0 & 1 & 1 \\
\hline & Problemas cardíacos & 0 & 1 & 1 \\
\hline & Problemas articulares & 1 & 6 & 7 \\
\hline & Falta de tempo & 0 & 2 & 2 \\
\hline & Total & 2 & 16 & 18 \\
\hline
\end{tabular}

No decorrer das ligações buscou se resgatar os conhecimentos prévios dos usuários acerca do tema, e sempre que surgiam dúvidas sobre o autocuidado da doença, os participantes foram estimulados a respondê-las caso soubessem. Percebemos que o monitoramento telefônico proporcionou a aquisição conhecimentos e reflexão sobre as práticas de autocuidado, a troca de experiência, fortalecimento do vínculo com os profissionais de saúde e o compartilhamento do sentimento.

Observamos que os usuários possuem a necessidade de falar sobre as suas dificulda- des das praticas de autocuidado e aproveitaram o momento do monitoramento para expressar seus sentimentos de angústia e desafio para a realização das praticas de atividade física e dieta. A maioria dos usuários mostrou se muito orientada quanto ao autocuidado da doença, relatando que apesar de não conseguir frequentar as atividades educativas na unidade de saúde, conseguem manter o acompanhamento das praticas de autocuidado.

Os conhecimentos sobre a doença, sentimentos, elaboração de metas e estímulo para o enfretamento das dificuldades encontradas 
foram discutidos entre bolsistas e usuários para que durante o monitoramento os mesmos pudessem refletir sobre as suas práticas de autocuidado. Os profissionais enfatizaram os sentimentos dos usuários em relação à doença, sendo destacados pelos participantes a raiva, a depressão, o medo da doença e o receio de usar insulina. A valorização dos sentimentos vivenciados e do comportamento dos usuários em relação à doença é uma resposta importante para a adesão e atitudes relacionadas ao autocuidado.

Esta estratégia educativa estimulou os usuários a assumirem uma postura ativa na elaboração do autocuidado associado à dieta e atividade física.

\section{DISCUSSÃO E CONCLUSÃO}

O estudo atual compõe o programa de educação em diabetes tipo 2, vem sendo desenvolvido desde 2007 em quatro Unidades Básicas de Saúde da cidade de Belo Horizonte, por uma equipe multidisciplinar (médicos, enfermeiros, fisioterapeuta, nutricionista, farmacêutico e terapeuta educacional), cujo objetivo é motivar os usuários com diabetes, e encorajá-los a aderirem ao regime terapêutico da doença, mediante a educação recebida, que está relacionada ao conhecimento e à mudança voluntária de comportamento para o cumprimento da educação do autogerenciamento dos cuidados. Outros estudos (9-11) têm sido realizados visando avaliar o desempenho desta metodologia.

O sistema de monitoramento via telefone pode ser considerado um método adjuvante no seguimento dos usuários com diabetes, uma vez que a sua utilidade está voltada ao reforço de um plano de educação para o autocuidado da doença. $\mathrm{O}$ método procurou focar em dois aspectos cruciais: a informação e comunicação em saúde para o autocuidado da doença.

O telefone é uma ferramenta tecnológica de fácil uso e é quase universalmente disponível no Brasil. Sendo assim, pode ser uma estratégia interessante de comunicação com usuários atendidos em um serviço público de saúde (10). O sistema de monitoramento por meio de ligações telefônicas reúne características potenciais de simplicidade, baixo custo e rapidez. No Brasil, esse sistema vem sendo utilizado com sucesso em populações nas quais os serviços de telefonia alcançam a grande maioria das residências, situação que pode ser projetada para a maioria das cidades brasileiras num prazo relativamente curto $(10,11)$.

O monitoramento foi uma das ações educativas realizadas de forma sistematizada a cada três meses (periodicidade), cuja finalidade consiste em melhorar os conhecimentos e as atitudes em relação à doença para o favorecimento da mudança de comportamento associada ao autogerenciamento dos cuidados e o controle da doença. Encontrase entre seus objetivos específicos, em relação aos usuários: melhorar os conhecimentos, no que diz respeito o diabetes, a dieta e a prática de exercícios físicos; tomar consciência dos valores e atitudes relacionados aos aspectos psicológicos associados à doença; favorecer a mudança de comportamento para a adesão a dieta e atividade física; melhorar o controle clínico da doença.

No monitoramento da educação do autocuidado em diabetes, observou-se que os usuários reconhecem a dieta e a prática regular de atividades físicas como fatores importantes para o controle da doença. No entanto, esses não foram relatados como hábitos praticados com freqüência pela maioria. A educação e a transferência de conhecimento é um processo difícil, especialmente no caso do diabetes, uma doença que afeta indivíduos de todas as idades, com diferentes graus educacionais e com diferentes bases sociais e ambientais (6).

Segundo a literatura (6-8), a construção de novos conhecimentos conduz à aquisição de comportamentos preventivos e estimula 
o indivíduo a compreender seus problemas e a escolher a solução apropriada para o gerenciamento dos cuidados da doença. Esse processo foi possível com os diabéticos que receberam atendimento por telefone, valorizando-se a troca dialógica com os profissionais de saúde/alunos.

A monitorização telefônica, por meio da escuta qualificada e conduzida de forma dialógica, permitiu potencializar as orientações para a educação do autocuidado, especialmente, dentro das áreas de nutrição e atividade física. Essa estratégia possibilitou maior disponibilidade para o acesso à comunicação, bem como a comodidade em receber as informações sobre a doença em domicílio. As limitações sobre o monitoramento por telefone foram em relação ao controle do tempo das ligações para alguns usuários, que, às vezes, sentiam necessidade de conversar sobre os conflitos familiares, sobre a depressão e sobre a doença.

A intervenção por via telefônica requer: empatia; capacidade de escuta; capacidade de transmitir informações de forma clara e objetiva; capacidade de adequar informações às necessidades apresentadas pelos usuários, capacidade de captar informações por meio da entonação da Voz (uma parte importante da escuta é perceber o que não é diretamente expresso); saber controlar o tempo da chamada; saber lidar com as chamadas difíceis, não se deixando manipular, repetindo a mesma informação; saber reconhecer quando e como terminar uma chamada, questionar qual o melhor horário para novo contato (24).

Esse instrumento de comunicação por telefone mostrou-se bastante efetivo em facilitar a interação profissional-indivíduo, confirmado pelas conversas descontraídas e até mesmo desabafos durante o monitoramento. A interação entre as alunas com os usuários também foi vista de forma efetiva, contribuindo para formação profissional e para a construção da visão dos usuários como alguém que tem vontades, necessidades e não somente como um doente.

O acesso, uso e apropriação para os usuários com telefone foi como uma tecnologia de informação e comunicação, alcançando um grande número de usuários em um tempo relativamente curto. Os benefícios desse tipo de intervenção a distância, abrangem a velocidade de acesso do usuário ao profissional de saúde; diminuição do tempo de espera para a consulta e redução do tempo e custo na locomoção dos usuários, além da possibilidade de aumentar a frequência dos contatos e de facilitar o retorno do usuário (8-10). Ainda, o monitoramento favorece a comunicação efetiva, a escuta e a compreensão entre o profissional de saúde e do usuário. Proporciona também a capacidade de negociação com os usuários, visando à redução das barreiras ao atendimento de qualidade para alcançar a educação em saúde de forma efetiva em diabetes (8).

Nesse cenário, a ligação telefônica surge como uma possibilidade única, em face da necessidade de superar distâncias e de acrescentar oportunidades de comunicação e troca $(9,12)$.

Os usuários relatavam grande satisfação por receber um telefonema, pois assim, sentiam-se acolhidos e motivados a continuar, demonstrando o quanto a atenção ao indivíduo é importante para a educação do autocuidado em diabetes. Além disso, o fato das ligações telefônicas serem realizadas alguns dias antes da data prevista para o processo educativo, evitava que os usuários esquecessem a data agendada, uma vez que o intervalo entre os grupos era de aproximadamente três meses.

A proposta de manter o mesmo aluno nos contatos com determinado usuário foi positiva, pois se percebeu que o vínculo foi se fortalecendo a cada contato e o usuário demonstrou satisfação em receber as ligações.

Assim sendo, a partir dos resultados obtidos, constatou-se que o telefone é um instrumento que serve como um meio que favorece a promoção da saúde, uma vez que 
permitiu um levantamento de dados úteis à elaboração de estratégias que possam guiar medidas educativas à usuários com diabetes mellitus tipo 2. Considera-se que a opção do programa educativo em diabetes pelo monitoramento via telefone favoreceu o compartilhamento de saberes.

O monitoramento telefônico como tecnologia de informação e comunicação permitiu fortalecer a educação dos usuários com diabetes, visando melhorar o controle do glicêmico. Além disso, fortaleceu o vínculo entre usuários e profissionais e possibilitou que os profissionais interviessem junto às dificuldades dos usuários no manejo da doença, de forma rápida e efetiva.

Em síntese o monitoramento via telefone apresentado é capaz de atingir boa performance, em termos de educação e comunicação para o autocuidado em diabetes. Entretanto, é preciso definir melhor a aplicabilidade, a sustentabilidade, a continuidade, e a aceitação da população e dos órgãos públicos e privados. Esse monitoramento poderia ser um veículo capaz de fornecer orientação aos usuários com diabetes sobre medidas de prevenção e controle da doença, como incentivar o autocuidado, a prática de hábitos saudáveis, como também evitar as complicações do diabetes.

Dentre os limites do estudo citamos o fato de tratar-se de estudo em usuários cadastrados nas quatro unidades, que se manifestaram pela adesão ao programa e que possuem telefone, não sendo, portanto, representativo do restante da população de diabéticos da cidade.

O monitoramento bem sucedido é aquele que estimula e incentiva um diálogo entre sujeitos ativos e reflexivos capazes de ativarem juntos e de forma autônoma mecanismos de mudança, permitindo, como no caso da educação em diabetes, maior autogerenciamento dos cuidados. Mudanças dependem da motivação dos agentes e são, assim, gradativas, reflexivamente orientadas de acor- do com o contexto em que os sujeitos estão envolvidos e onde os hábitos são moldados. Nessa medida, o sucesso da intervenção por telefone e de promoção da saúde depende de que as estratégias propostas sejam adequadas ao contexto sócio-cultural em que os sujeitos tomam decisões. Um monitoramento bem sucedido é aquele que se a ajusta e se afina ao contexto em que representações, comportamentos e necessidades dos sujeitos são moldados.

\section{AGRADECIMENTOS}

Pesquisa Financiada pela BRIDGES. "BRIDGES is an IDF program supported by an educational grant from Lilly Diabetes" e Fundação de Amparo a Pesquisa de Minas Gerais. FAPEMIG, apoiada pelo Núcleo de Gestão, Educação e Avaliação em Saúde do Departamento de Enfermagem Aplicada da Escola de Enfermagem da Universidade Federal de Minas Gerais (EE/UFMG).

\section{REFERÊNCIAS}

1. Ministério da Saúde (Brasil), Secretaria de Vigilância em Saúde, Departamento de Análise de Situação em Saúde. Saúde Brasil 2008: uma análise da situação de saúde no Brasil. Brasília: Ministério da Saúde, 2009.

2. Malta DC, Cezário AC, Moura L de. A construção da vigilância e prevenção das doenças crônicas não transmissíveis no contexto do Sistema Único de Saúde. Epidemiol. Serv. Saúde. 2006 ; 15(3): 4765.

3. Torres HC, Hortale VA, Schall V. A experiência de jogos em grupos operativos na educação em saúde para diabéticos. Cad Saude Publica. 2003; 19(4): 10391047. 
4. Chaves EC, Oyama SMR. Abordagem telefônica como estratégia para promoção da saúde. Rev Gaucha Enferm. 2007; 28 (2): 171-179.

5. Valenzuella JI, Camacho JC, Argüello A, Cendales JG, Fajardo R. Percepciones de los trabajadores del sector salud frente a Internet y lãs tecnologias móviles en Colombia. Rev Panam Salud Publica. 2009; 25(4): 367-374.

6. Bernal R, Silva NN. Cobertura de linhas telefônicas residenciais e vícios potenciais em estudos epidemiológicos. Rev Saude Publica. 2009; 43(3): 421-426.

7. Freire P. Pedagogia do Oprimido. $17^{\mathrm{a}}$ ed. Rio de Janeiro, editora Paz e Terra, 1987.

8. Lange I, Campos S, Urrutia M, Bustamante C, Alcayaga C, Tellez A et al. Efecto de un modelo de apoyo telefónico en el auto-manejo y control metabólico de la Diabetes tipo 2, en un Centro de Atención Primaria, Santiago, Chile. Rev Med Chile. 2010; 138(6): 729-37.
9. Martínez A. Autopercepción de la enfermedad en pacientes diagnosticados de diabetes mellitus tipo 2 que acuden a consulta de enfermería. Enfermería Global. 2012; 11(3).

10. Bustamante C, Riquelme G, Alcalaya C, Lange I, Urrutia M . Experiencia de transferencia tecnológica de la Consejería Telefónica para Cuidados Crónicos de Salud, a equipos de Atención Primaria. Enfermería Global. 2012; 11(3).

11. Torres HC, Franco L, Stradioto M, Hortale V, Shall V. Avaliação estratégica de educação em grupo e individual no programa educativo em diabetes. Rev Saude Publica. 2009; 43(2): 291-298.

12. Malta DC, Moura EC, Castro AM, Cruz DKA, Morais Neto OL, Monteiro CA. Padrão de atividade física em adultos brasileiros:resultados de um inquérito por entrevistas telefônicas, 2006. Epidemiol. Serv. Saúde. 2009; 18(1): 7-16. 